Aliment Pharmacol Ther. 2020 January ; 51(1): 149-157. doi:10.1111/apt.15538.

\title{
Vedolizumab use is not associated with increased malignancy incidence: GEMINI LTS study results and post-marketing data
}

\author{
Timothy Card ${ }^{1}$, Ryan Ungaro ${ }^{2}$, Fatima Bhayat ${ }^{3}$, Aimee Blake $^{3}$, Gary Hantsbarger $^{3}$, Simon \\ Travis ${ }^{4}$ \\ ${ }^{1}$ Faculty of Medicine and Health Sciences, University of Nottingham, Nottingham, UK \\ ${ }^{2}$ The Henry D. Janowitz Division of Gastroenterology, Icahn School of Medicine at Mount Sinai, \\ New York, NY, USA \\ ${ }^{3}$ Takeda Pharmaceuticals International Co., Cambridge, MA, USA \\ ${ }^{4}$ Translational Gastroenterology Unit, NIHR Oxford Biomedical Research Centre, Oxford \\ University Hospitals NHS Foundation Trust, Oxford, UK
}

\section{Summary}

Background: Vedolizumab is a gut-selective antibody to $\alpha_{4} \beta_{7}$ integrin approved to treat moderate-to-severe Crohn's disease and ulcerative colitis in adults. Inflammatory bowel disease (IBD) and immunosuppressant use are associated with increased risk of malignancy.

\begin{abstract}
Aim: To analyse the incidence of malignancy with vedolizumab treatment in the GEMINI longterm safety (LTS) study and post-marketing (PM) setting.

Methods: Malignancy data from the LTS study (May 2009 to May 2018), and data from the vedolizumab Global Safety Database (20 May 2014 to 19 May 2018), were identified using Medical Dictionary for Regulatory Activities coding. The number of patients experiencing malignancies in the LTS study (excluding malignancies within 1 year following vedolizumab initiation) was indirectly standardised against the number expected, using age- and sex-specific rates in patients with IBD from Optum's Clinformatics ${ }^{\mathrm{TM}}$ Data Mart (CDM) database.
\end{abstract}

Results: Among 1785 patients with $\geq 1$ year of follow-up post-vedolizumab initiation in the LTS study (total 5670 patient-years), observed numbers of malignancies were similar to those expected compared with CDM data (31 vs 29 ; ratio of observed to expected events $=1.08 ; P=0.71 ; 95 \%$ confidence intervals $[\mathrm{CI}] 0.73,1.53)$. The most common malignancies were renal and bladder (6). PM, 293 patients reported 299 malignancies (including malignancies within 1 year following

\footnotetext{
Correspondence: Dr Timothy Card, Faculty of Medicine and Health Sciences, University of Nottingham, Nottingham, UK. tim.card@nottingham.ac.uk. AUTHORSHIP

Guarantor of the article: Dr Fatima Bhayat.

Author contributions: All authors made substantial contributions in the conception and design of the study, or acquisition of data, or analysis and interpretation of data; in drafting the article or revising it critically for important intellectual content; and gave final approval of the version submitted.

SUPPORTING INFORMATION

Additional supporting information will be found online in the Supporting Information section at the end of the article.
} 
vedolizumab initiation), in approximately 208050 patient-years of vedolizumab exposure. Lower gastrointestinal malignancies were most common (59).

Conclusions: The number of malignancies in the LTS study was similar to that expected from an IBD population with no statistically significant differences, although few confounders could be corrected for. Limitations of PM safety reporting require consideration; however, the number of malignancies with vedolizumab appeared low.

\section{1| INTRODUCTION}

In patients with Crohn's disease (CD), it has been estimated that the risk of colorectal, upper gastrointestinal (GI) and small bowel cancers is increased 1.9-2.5-fold, 2.9-fold and 27.1fold, respectively, vs the background population. ${ }^{1,2}$ In patients with ulcerative colitis (UC), the risk of colorectal and hepatobiliary cancers is estimated to be 2.4-fold and 2.6-fold higher, respectively, than the general population. ${ }^{2,3}$ No unequivocal excess risk of extraintestinal cancer has been established in patients with CD or UC; however, one study found increased risk at specific anatomical sites, including lungs, urinary bladder and skin, in patients with $\mathrm{CD}$, and increased risk of leukaemia in patients with $\mathrm{UC}$, but no significant increase overall. ${ }^{2}$ A further study found increased lymphoma rates in men with $C D$ vs healthy controls. ${ }^{4}$ However, estimates were not adjusted for relevant risk factors, such as smoking or previous treatment exposure, in either study.

In addition to the impact of underlying disease, it is important to consider the possibility of increased risk of malignancy associated with treatments intended to reduce GI tract inflammation in CD or UC. Immunomodulators, including thiopurines and biologics (such as infliximab and other anti-tumour necrosis factor alpha [TNFa] antibodies), are commonly used to treat Moderate-to-severe active disease. ${ }^{5}$ Many treatments have been associated with increased malignancy risk in patients with inflammatory bowel disease (IBD), notably thiopurines; for example, one meta-analysis found a 4.9-fold increase in the incidence of lymphoma. ${ }^{6}$ Notably, infliximab carries a "black box" warning regarding malignancies. ${ }^{7}$ Furthermore, a population-based cohort study found that long-term (>12 months) immunesuppression was associated with increased risk of haematologic cancer, non-Hodgkin lymphoma, squamous cell skin cancer and overall cancer, primarily attributable to thiopurine use. ${ }^{8}$ In contrast, a registry-based cohort study found no significant increase in cancer risk for patients with IBD receiving anti-TNFa therapy vs no anti-TNFa therapy over a median follow-up of 3.7 years. ${ }^{9}$

Vedolizumab is a gut-selective antibody to $\alpha_{4} \beta_{7}$ integrin approved to treat moderate-tosevere active CD or UC in adults. ${ }^{10-12}$ Integrated safety data from six vedolizumab trials have shown a low incidence of malignancy, ${ }^{13}$ although trial follow-up periods and patient numbers were insufficient to evaluate this fully. Since first approval, additional safety data reflecting longer-term use of vedolizumab have been collected in an open-label extension study (GEMINI long-term safety [LTS] study; ) ${ }^{14,15}$ and the vedolizumab Global Safety Database (GSDB), through individual case safety reports from post-marketing (PM) sources. Given the importance of understanding malignancy risk associated with IBD treatments, this 
analysis aims to provide further evidence on whether there is an increased incidence of malignancy in patients with CD or UC receiving vedolizumab.

\section{2 | MATERIALS AND METHODS}

\subsection{GEMINI LTS study data}

Safety data for vedolizumab were collected in the LTS study between May 2009 and May 2018. Most patients were recruited from the GEMINI 1, GEMINI 2 and GEMINI 3 trials, and an open-label phase 2 trial (); all assessed the efficacy and/or safety of vedolizumab in patients with CD or UC. Notably, the associated GEMINI inclusion criteria stipulated that patients with a prior history of malignancy were not eligible for participation. These trials, and the LTS study (using a June 2013 data cut-off), have been described previously. ${ }^{13-18}$ In the LTS study, patients randomised to vedolizumab in GEMINI 1, GEMINI 2 and GEMINI 3 continued to receive vedolizumab, whereas patients randomised to placebo switched to vedolizumab. The remaining patients, who enrolled directly into the LTS study, were vedolizumab-naïve before treatment. Notably, patients received 4-weekly infusions of vedolizumab, compared with the usual dosing regimen of 8-weekly infusions as described in the vedolizumab prescribing information. ${ }^{12}$ Patients with family or personal history of colorectal cancer, those aged $>50$ years, or those with another known risk factor for colorectal cancer were only included in the LTS study if their colorectal cancer surveillance was up-to-date.

The LTS study was conducted in accordance with the ethical principles in the Declaration of Helsinki, and all appropriate study documentation was reviewed by the Institutional Review Board and independent ethics committee according to local regulations. All rollover patients signed consent forms before enrolment; directly enrolled patients signed consent forms before commencement of any study-related procedures. All authors had access to the relevant study data, and reviewed and approved the final manuscript.

2.1.1 Observed malignancy events-This report describes all data regarding malignancies gathered from the LTS study until 19 May 2018, and includes all patients who received at least one dose of vedolizumab by this date. Relevant adverse events (AEs) were identified using Medical Dictionary for Regulatory Activities (MedDRA) version 20.0 System Organ Class terms relating to neoplasms (excluding benign). To avoid misclassifying prevalent malignancy, patients with a diagnosis of malignancy prior to, or within 1 year after starting treatment with vedolizumab were excluded, as were the associated follow-up time and the following events: benign neoplasms, basal cell carcinomas, colon adenomas, haemangioma, neurilemmoma, non-melanoma skin cancers and malignancies with diagnoses reported before vedolizumab initiation. Total exposure time in the analysis for patients enrolled in the LTS study was measured from 1 year after the date of first vedolizumab infusion in the LTS study or lead-in study until the last recorded assessment date for the patient, based on visit dates, dates of infusions and dates of AEs. Identified malignancies were categorised by anatomical site (Table S1 provides grouping of MedDRA preferred terms). Malignancies were also split by indication for vedolizumab $(\mathrm{CD} / \mathrm{UC})$, previous immunomodulator exposure (yes/no; including anti-TNFa therapy) and disease 
duration ( 5 years/ $>5$ years; defined as the time between disease diagnosis and vedolizumab initiation).

2.1.2 Expected malignancy events-For comparison with observed malignancy rates in the LTS study, expected numbers of malignancy events in patients with IBD were calculated using indirect standardisation, with patients with IBD in the US Optum's Clinformatics ${ }^{\mathrm{TM}}$ Data Mart (CDM) database as a reference population. The CDM database is a commercial and Medicare de-identified claims database of almost 48 million members. An advantage of the CDM database is that many other databases only cover commercial or Medicare supplemental plans. The CDM database contains claims from June 2000 to December 2017, with diagnoses recorded using International Statistical Classification of Diseases and Related Health Problems, 9th Revision (ICD-9) and ICD-10 codes.

Individuals in the CDM database with a diagnosis of $\mathrm{CD}$ or $\mathrm{UC}$ were identified as those with at least two different claims associated with CD or UC diagnostic codes (ICD-9: 556.x or 555.x; ICD-10: K51.x [CD], K50.x [UC]) 230 days apart. Patients with diagnoses of both $\mathrm{CD}$ and UC were classified as having "unspecified IBD" and excluded. Malignancies in the CDM database were identified using ICD-9 and ICD-10 codes for malignant neoplasms (ICD-9: 140.x to 209.x; ICD-10: C00.x to C96.x). To align diagnoses from the CDM database as closely as possible with those of interest in the LTS study, benign neoplasms, neoplasms of uncertain behaviour, in situ neoplasms and all skin neoplasms, except malignant melanomas, were excluded. To mirror exclusions in the exposed cohort from the LTS study, patients with a diagnosis of malignancy recorded within the database at any point prior to 1 year after IBD diagnosis were excluded from the CDM population. The start date for the calculation of patient-time in the CDM population was assigned as the date 1 year after IBD diagnosis, so that as in the LTS study, the first year of available follow-up was censored to avoid misattribution of prevalent malignancies as incident. Following identification of relevant malignancies, patient-years of follow-up were estimated. In this calculation, index date was defined as the date of the first recorded ICD code of CD or UC. If a patient had at least 1 year of follow-up after the IBD index date and did not have a relevant malignancy diagnosis, patient-years were calculated using the following formula:

Number of patient-years $=(($ last enrolment date - IBD index date +1$) / 365.25)-1$

If a patient had a relevant malignancy diagnosis and at least 1 year of follow-up, patientyears were calculated using the following formula:

Number of patient-years $=(($ malignancy diagnosis date-IBD index date +1$) / 365.25)-1$

Using the number of patient-years, rates of malignancy in the reference population were calculated for each age (18-39, 40-44, 45-49, 50-54, 55-59, 60-64, 65-69, 70-74 and 75+ years old) and sex stratum by dividing the numbers of events in each stratum by the total number of patient-years. The resulting rates in the reference population for each age stratum were then multiplied by the total follow-up time for the LTS study population to provide estimates of the age- and sex-adjusted expected numbers of malignancies for each 
anatomical site. Finally, the ratio of observed to expected events was calculated, with $95 \%$ confidence intervals (CIs) and hypothesis tests of equal event rates in the reference and study population conducted based on a Poisson distribution of the number of observed events.

\subsection{PM data}

All PM AE reports received by the licence holder, Takeda Pharmaceutical Company Ltd, since vedolizumab approval are held in the vedolizumab GSDB. Report sources include: spontaneous reports from patients, healthcare professionals and regulatory authorities; solicited reports from patient-support and market-research programmes; and reports extracted from the literature. All PM reports concerning malignancies received between vedolizumab approval and 19 May 2018 were identified using the MedDRA version 21.0 System Organ Class term "neoplasms benign, malignant, and unspecified (including cysts and polyps)". For consistency with LTS study data, benign neoplasms, colon adenomas and all malignancies with diagnoses reported before vedolizumab initiation were excluded; however, events reported within the first year of vedolizumab exposure were included as a significant number of reported malignancies did not contain information on the malignancy diagnosis date. Relevant reports identified were then categorised by anatomical site of malignancy (Table S2), and by indication (CD, UC, unspecified IBD, other or not reported). Patient-years of follow-up were considered equivalent to total dispensed vedolizumab/annual recommended vedolizumab dose.

\section{3 | RESULTS}

\subsection{GEMINI LTS study}

3.1.1 Overview of events and baseline characteristics-In total, 2243 patients were enrolled into the GEMINI LTS study by the 19 May 2018 data cut-off, with a median follow-up of 1014 days (2.8 years), generating 7746 patient-years of data. Of these, 1822 patients had previously participated in the phase $2(\mathrm{n}=37)$, GEMINI $1(\mathrm{n}=675)$, GEMINI 2 $(\mathrm{n}=726)$ or GEMINI $3(\mathrm{n}=384)$ trials. The remaining 421 patients were vedolizumabnaïve and enrolled directly into the LTS study. Of the 2243 patients, 1785 had $\geq 1$-year follow-up after vedolizumab initiation. In 5670 patient-years of included vedolizumab exposure, 31 patients experienced malignancies of interest, corresponding to $2 \%$ of patients enrolled in the LTS study with follow-up $\geq 1$ year after first infusion.

When comparing baseline characteristics of the 1785 patients with follow-up $\geq 1$ year after vedolizumab initiation with those of the entire LTS study population (including those with follow-up exclusively <1 year after vedolizumab initiation; $N=2243$ ), there were no substantial differences. Of the 1785 patients with follow-up $\geq 1$ year after vedolizumab initiation, 880 (49\%) were female, vs $14(45 \%)$ of the 31 patients with malignancies (Table 1). The mean (SD) age in the LTS study was 39.5 (12.96) years, compared with 51.2 (10.50) years for patients experiencing a malignancy. Regarding indication, $1034(58 \%)$ had CD and $751(42 \%)$ had UC, while 17 (55\%) and 14 (45\%) patients with a malignancy had CD and UC respectively. Prior use of anti-TNFa therapy was reported by $62 \%$ of patients with CD and $43 \%$ of patients with UC in the LTS study; in patients experiencing a malignancy, these proportions were $65 \%$ and $57 \%$ respectively. 
Malignancies occurring in the highest number of patients were renal and bladder $(n=6 ; 3$ $\mathrm{CD}, 3 \mathrm{UC}$ ), lower GI ( $\mathrm{n}=5 ; 2 \mathrm{CD}, 3 \mathrm{UC}$ [including one patient with UC who experienced two events]) and respiratory ( $\mathrm{n}=4 ; 1 \mathrm{CD}, 3 \mathrm{UC}$; Table 2). Malignancies at all other sites were experienced by three or fewer patients, and malignancy numbers at each site were generally similar between patients with CD and those with UC.

\subsubsection{Malignancies by previous immunomodulator exposure (including anti- TNFa agent exposure) and disease duration-In 1293 patient-years of data on} patients without prior immunomodulator exposure, three patients experienced a malignancy (Table 2), corresponding to 232 events per 100000 patient-years. In 4377 patient-years of data on patients with prior immunomodulator exposure, 28 experienced a malignancy (Table 2), corresponding to 640 events per 100000 patient-years.

In 2196 patient-years of data on patients with disease duration of $\mathbf{5}$ years, eight patients experienced a malignancy, corresponding to 364 events per 100000 patient-years. In 3474 patient-years of data on patients with disease duration $>5$ years, 23 experienced a malignancy, corresponding to 662 events per 100000 patient-years.

3.1.3 Comparison of observed vs expected malignancy events-The overall number of patients with malignancies observed in the LTS study $(\mathrm{n}=31)$ was similar to the expected number estimated using an age- and sex-adjusted reference population $(\mathrm{n}=29 ; P=$ 0.71 ), corresponding to a ratio of observed to expected events across all anatomical sites of 1.08 (95\% CI, 0.73-1.53; Figure 1). With respect to individual malignancy sites, observed numbers of malignancies did not differ significantly from the expected number at any site (Figure 1).

\section{2 | PM setting}

3.2.1 Overview of events and baseline characteristics-In the context of approximately 208050 patient-years of vedolizumab exposure in the PM setting, 299 malignancy events in 293 patients were identified from the vedolizumab GSDB, corresponding to $0.4 \%$ of all reported AEs $(\mathrm{N}=80218)$ and approximately 144 events per 100000 patient-years; this figure is placed in context in the discussion. Six patients reported a malignancy at more than one site. Of the malignancies reported, 126 (42\%) did not contain information on malignancy diagnosis date relative to vedolizumab therapy initiation, and 128 malignancies $(43 \%)$ were reported to occur within 1 year after starting vedolizumab treatment. The other 45 malignancies $(15 \%)$ occurred $\geq 1$ year after vedolizumab initiation.

Of the 293 patients with reported malignancies, 145 (49\%) were female and $160(55 \%)$ were aged 18-64 years (Table 3). Regarding indication, 129 patients (44\%) had CD and 116 (40\%) had UC. Prior and/or concomitant anti-TNFa therapy, concomitant corticosteroid use and/or other concomitant immunomodulatory therapies were reported in $47 \%, 29 \%$ and $20 \%$ of patients, respectively (Table 3); $24 \%$ of patients did not report using these treatments prior to, or concomitantly with, vedolizumab.

3.2.2 Malignancies by anatomical site and indication-Across all indications, the most common malignancies were of the lower GI tract and lymphoma (59 [20\%] and 33 
[11\%] events respectively). An unspecified malignant neoplasm was reported in 18 patients (6\%), and a GI neoplasm of "unspecified" status was reported in one further patient $(0.3 \%)$, which was included in this analysis because there was insufficient information to determine if the neoplasm was benign or malignant.

Numbers of reported malignancies were similar whether patients were receiving vedolizumab for $\mathrm{CD}$ or UC (134 and 117 respectively). Lower GI malignancies were the most commonly reported malignancy in patients with CD or UC (21 and 34 respectively).

\section{4 | DISCUSSION}

In the LTS study, the number of observed malignancies with vedolizumab was similar to the reference population with IBD from the CDM database (ratio of observed to expected, 1.08; $P=0.71$ ). It must be acknowledged that the standardisation performed had limitations. Although sex- and age-specific rates were used and patients with a history of malignancy prior to starting treatment for vedolizumab, or with a diagnosis of malignancy within 1 year following vedolizumab initiation, were excluded, other confounders, such as disease duration and receipt of previous immunomodulators, could not be adjusted for. The importance of this is highlighted by the fact that 28 of the 31 patients with a malignancy reported previous immunomodulator use and 23 of 31 had a disease duration of $>5$ years.

In the PM setting, malignancies were reported in 293 patients in 208050 patient-years (approximately 144 events per 100000 patient-years), indicating no signal of increased risk of malignancy in patients with IBD receiving vedolizumab in the real-world setting.

However, this should be interpreted in the context of the inherent biases of PM data sources, including that voluntary reporting means not all malignancy events are necessarily captured. Crude comparisons suggest the rate in the PM setting is substantially lower than reported from other sources, including the LTS study (approximately 547 events per 100000 patientyears), and by Bernstein et $\mathrm{al}^{4}$ in a study linking a population-based IBD database to a comprehensive cancer registry to determine cancer incidence among a North American population with IBD between 1984 and 1997 (690 events per 100000 patient-years). In addition, because $47 \%$ of patients reporting malignancies in the current study had prior or concomitant exposure to anti-TNFa agents and $20 \%$ reported concomitant use of other immunomodulatory therapies, prior or concomitant treatment may have contributed to their risk of malignancy.

The most common sites of malignancy in the LTS study were renal and bladder, lower GI, and respiratory. The observed numbers of malignancies at these sites were not significantly higher than expected based on the CDM reference population with IBD ( $P>0.05$ for each). However, patient numbers were no greater than six for any malignancy site, hence there was very limited power to detect any relationship to an individual malignancy. In the PM setting, the most frequently reported cancers were GI (21 and 34 events in patients with CD or UC respectively). These cancers are well documented in patients with IBD, ${ }^{19}$ and are reported to be the most frequently occurring malignancies in this population. ${ }^{1-4}$ 
In the LTS study, the number of patients with lower GI malignancies $(\mathrm{n}=5)$ was similar to the number expected based on indirect standardisation against the CDM reference population (ratio, 1.06; $P=1.00$ ). However, the low number of events, and therefore limited power, again requires consideration when interpreting the result. In the PM setting, the unadjusted rate of lower GI malignancies equated to 28.4 per 100000 patient-years. In the Surveillance, Epidemiology and End Results (SEER) registry, overall age-adjusted incidence of colorectal cancers in the general population was 40.1 per 100000 people per year. ${ }^{20} \mathrm{In}$ studies of patients with IBD, Bernstein et al found overall incidences of 146 and 44 per 100 000 person-years for colon and rectal cancer, respectively, ${ }^{4}$ and Söderlund et al reported an overall incidence of 95 cases of colorectal cancer per 100000 person-years. ${ }^{21}$ Although these datasets incorporated preferred terms relating to slightly different sites than considered in the PM setting reported here, and we have not standardised these figures for age and sex, incidence of lower GI malignancies seen with vedolizumab in the PM setting appears low.

These data reflect large numbers of patient-years from the LTS study and PM setting. The LTS study used stringent eligibility criteria, and recorded detailed, consistent data on events, patient characteristics and treatment histories. ${ }^{22}$ However, there are a number of limitations that should be acknowledged.

This study included a relatively small proportion of patients, who consented to involvement in the clinical trial with strict eligibility criteria, so results may be less generalisable to realworld setting patients. Our analysis also used a patient population with IBD from the CDM database as a reference population. However, our methodology of indirect standardisation does not allow correction for all confounders (eg differences in IBD severity, duration, activity and extent of colitis, and medication history), although we adjusted for the effects of age and gender. On the other hand, as biologic medications such as vedolizumab are likely to be given to patients with disease of greater than average severity, it is the authors' belief that, were these data available to allow correction, the expected number of malignancies would likely increase rather than reduce. Therefore, we do not believe the use of the CDM database as a reference population invalidates the findings of this study.

Although, where a history of malignancy prior to starting IBD treatment or a diagnosis of malignancy within 1 year following treatment initiation were recorded, patients were excluded, the CDM database lacks full medical histories of patients, and thus only events occurring after joining the database are recorded. Cancer diagnoses made prior to inclusion in the database are therefore not recorded and patients may not be excluded appropriately, which could bias the number of cases identified. In addition, disease severity of patients in the CDM database (all IBD severities) may not reflect that of patients in the LTS study (moderate-to-severe active IBD). However, as no precisely comparable IBD population of patients not treated with vedolizumab was available, we considered the CDM reference population the most suitable for this analysis to which we could gain access and so chose to accept this bias.

Another common source of comparators in such studies is a high-quality general population malignancy database such as the SEER registry. ${ }^{23}$ However, the SEER registry includes general, rather than IBD-specific population data, and consequently reflects the risks of 
malignancy in the general population, which are lower than those of IBD patients. ${ }^{1-3}$ Furthermore, as the SEER registry provides data on cancer incidence in the general population, it does not easily allow for the generation of rates excluding those with previous malignancy or with malignancy diagnoses within 1 year of treatment initiation, as we have attempted.

There were substantially more patient-years of exposure in the PM setting than in the LTS study, and these data more closely reflect vedolizumab use in real-world clinical practice. The vedolizumab GSDB includes important patient information (eg age, treatment history and indication), but this was incomplete for some patients. In addition, although most malignancies were new events, a small number may have resulted from exacerbations or metastases of malignancies reported in the GEMINI trials. Reporting to the vedolizumab GSDB is voluntary, meaning that the number reported here is likely to be lower than if all malignancies in the PM setting were reported. However, bias, such as increased reporting of more serious events (including malignancies), associated with PM data should be considered. There are also multiple confounding factors that may affect the risk of malignancy in patients with IBD, including disease duration, age at diagnosis, extent of disease, inflammation severity, treatment history, history of malignancy and smoking history, which are incomplete in some PM data reports. ${ }^{19}$ Finally, the duration of vedolizumab exposure in these patients is still relatively short.

In conclusion, the LTS study demonstrated that the total number of observed malignancies was similar to that expected after standardising against patients with IBD in the CDM database, with patients in the LTS study receiving 4-weekly infusions of vedolizumab as opposed to the usual 8-weekly infusion regimen. The significance of malignancy rates at specific sites should not be overinterpreted because of low numbers of events observed. Allowing for limitations of PM safety reports, the number of malignancies with vedolizumab use appeared low. Safety data on malignancy incidence from the LTS study and PM setting suggest that the favourable malignancy-related safety profile of vedolizumab in $\mathrm{CD}$ and UC observed in preapproval clinical trials is retained with longer-term use. Although limitations of these data sources must be considered, these data show no evidence of any significant increase in incidence of malignancy in patients with IBD receiving vedolizumab. Additional data on incidence of malignancy with vedolizumab use will be available from the post-authorisation safety study (PASS). PASS is a prospective, observational trial which is currently being conducted at multiple centres worldwide (). It aims to examine the long-term safety of vedolizumab vs other biologic agents in approximately 5000 participants with CD or UC (with a follow-up period of 7 years). Data from this study will supplement those from continued PM surveillance.

\section{Supplementary Material}

Refer to Web version on PubMed Central for supplementary material.

\section{ACKNOWLEDGEMENTS}


University received any payment financial or in kind related to this work. He has no active conflict of interest to declare but was formerly married to an employee of Takeda. Simon Travis is employed by Oxford University Hospitals NHS Foundation Trust and the University of Oxford. He has received grants or research support from AbbVie, Bühlmann, International Organization for the Study of Inflammatory Bowel Diseases, Lilly, MEDiadd, Norman Collison Foundation, UCB and Vifor; consulting fees from AbbVie, Ajinomoto, Almirall, Amgen, Asahi, Atlantic, Bioclinica, Biogen, Boehringer Ingelheim, Bristol-Myers Squibb, Calcico, Celgene, ChemoCentryx, Cosmo, Covance, Enterome, Falk, Ferring, Genentech, Gilead, Giuliani SpA, GlaxoSmithKline, Glenmark, Grünenthal, Immunocore, Immunometabolism, Istesso, Janssen, Lexicon, Lilly, Medarex, Merck, MSD, Napp, Neovacs, Novartis, Novo Nordisk, NPS Pharmaceuticals, Ocera, Otsuka, Pfizer, Pharm-Olam, Phillips, Proximagen, Quintiles, Receptos, Robarts, Roche, Sandoz, Shire, Sensyne, Sigmoid Pharma, Takeda, Theravance, TiGenix, Topivert, UCB, Vertex, VHsquared, Vifor, Warner Chilcott and Zeria; and speaker fees from AbbVie, Amgen, Biogen, Ferring, Sandoz, Shire, Takeda and Zeria. He has no stocks or share options. Ryan Ungaro has served as a consultant and/or advisory board member for Eli Lilly, Janssen, Pfizer and Takeda. He has received research support from AbbVie, Boehringer Ingelheim and Pfizer. He is supported by Career Development Awards from the Crohn's and Colitis Foundation and the National Institutes of Health (K23KD111995-01A1). The authors thank Baoguo Jiang, who provided statistical analysis support for this manuscript. Baoguo Jiang is an employee of Takeda Pharmaceuticals Inc

Funding information

This study was sponsored by Takeda Pharmaceutical Company Ltd. This work was supported in part by the National Institute for Health Research (NIHR) Oxford Biomedical Research Centre (BRC). The views expressed are those of the author(s) and not necessarily those of the NHS, the NIHR or the Department of Health. Medical writing assistance was provided by Fraser Harris and Alexandra Kisbey of Oxford PharmaGenesis Ltd, Oxford, UK, and was funded by Takeda Pharmaceutical Company Ltd.

\section{REFERENCES}

1. Jess T, Gamborg M, Matzen P, Munkholm P, Sorensen TI. Increased risk of intestinal cancer in Crohn's disease: a meta-analysis of population-based cohort studies. Am J Gastroenterol. 2005;100:2724-2729. [PubMed: 16393226]

2. Pedersen N, Duricova D, Elkjaer M, Gamborg M, Munkholm P, Jess T. Risk of extra-intestinal cancer in inflammatory bowel disease: meta-analysis of population-based cohort studies. Am J Gastroenterol. 2010;105:1480-1487. [PubMed: 20332773]

3. Jess T, Rungoe C, Peyrin-Biroulet L. Risk of colorectal cancer in patients with ulcerative colitis: a meta-analysis of population-based cohort studies. Clin Gastroenterol Hepatol. 2012;10:639-645. [PubMed: 22289873]

4. Bernstein CN, Blanchard JF, Kliewer E, Wajda A. Cancer risk in patients with inflammatory bowel disease: a population-based study. Cancer. 2001;91:854-862. [PubMed: 11241255]

5. Moss AC. Optimizing the use of biological therapy in patients with inflammatory bowel disease. Gastroenterol Rep (Oxf). 2015;3:63-68. [PubMed: 25567472]

6. Kotlyar DS, Lewis JD, Beaugerie L, et al. Risk of lymphoma in patients with inflammatory bowel disease treated with azathioprine and 6-mercaptopurine: a meta-analysis. Clin Gastroenterol Hepatol. 2015;13:847-858.e4. Quiz e48-58. [PubMed: 24879926]

7. Janssen Biotech Inc. REMICADE (infliximab) Prescribing Information 2018 http:// www.janssenlabels.com/package-insert/product-monograph/prescribing-information/REMICADEpi.pdf. Accessed December 2018.

8. van den Heuvel T, Wintjens D, Jeuring S, et al. Inflammatory bowel disease, cancer and medication: cancer risk in the Dutch population-based IBDSL cohort. Int J Cancer. 2016;139:1270-1280. [PubMed: 27170593]

9. Nyboe Andersen N, Pasternak B, Basit S, et al. Association between tumor necrosis factor-alpha antagonists and risk of cancer in patients with inflammatory bowel disease. JAMA. 2014;311:24062413. [PubMed: 24938563]

10. Takeda Pharmaceutical Company Ltd. ENTYVIO® (vedolizumab) Prescribing Information 2014 http://general.takedapharm.com/content/file.aspx? FileTypeCode=ENTYVIOPI\&cacheRandomizer=ab87b2b4-1e26-40e1-a77a-a7db84f28d9d. Accessed November 2018. 
11. European Medicines Agency (EMA). Vedolizumab, summary of product characteristics. https:// www.ema.europa.eu/documents/product-information/entyvioepar-product-information_en.pdf. Accessed November 2018.

12. Food Drug Administration (FDA). Vedolizumab, Prescribing Information. https:// www.accessdata.fda.gov/drugsatfda_docs/label/2014/125476s000lbl.pdf. Accessed November 2018.

13. Colombel J- F, Sands BE, Rutgeerts P, et al. The safety of vedolizumab for ulcerative colitis and Crohn's disease. Gut. 2017;66:839-851. [PubMed: 26893500]

14. Loftus EV Jr, Colombel JF, Feagan BG, et al. Long-term efficacy of vedolizumab for ulcerative colitis. J Crohns Colitis. 2017;11:400-411. [PubMed: 27683800]

15. Vermeire S, Loftus EV Jr, Colombel JF, et al. Long-term efficacy of vedolizumab for Crohn's disease. J Crohns Colitis. 2017;11:412-424. [PubMed: 27683798]

16. Feagan BG, Rutgeerts P, Sands BE, et al. Vedolizumab as induction and maintenance therapy for ulcerative colitis. N Engl J Med. 2013;369:699-710. [PubMed: 23964932]

17. Sandborn WJ, Feagan BG, Rutgeerts $\mathrm{P}$, et al. Vedolizumab as induction and maintenance therapy for Crohn's disease. N Engl J Med. 2013;369:711-721. [PubMed: 23964933]

18. Sands BE, Feagan BG, Rutgeerts $P$, et al. Effects of vedolizumab induction therapy for patients with Crohn's disease in whom tumor necrosis factor antagonist treatment failed. Gastroenterology. 2014;147:618-627.e3. [PubMed: 24859203]

19. Dyson JK, Rutter MD. Colorectal cancer in inflammatory bowel disease: what is the real magnitude of the risk? World J Gastroenterol. 2012;18:3839-3848. [PubMed: 22876036]

20. Howlader N, Noone AM, Krapcho M SEER cancer statistics review, 1975-2009 Vintage 2009 populations Bethesda MD: National Cancer Institute; 2009:2012.

21. Söderlund S, Brandt L, Lapidus A, et al. Decreasing time-trends of colorectal cancer in a large cohort of patients with inflammatory bowel disease. Gastroenterology. 2009;136:1561-1567. [PubMed: 19422077]

22. Day RO, Williams KM. Open-label extension studies: do they provide meaningful information on the safety of new drugs? Drug Saf. 2007;30:93-105. [PubMed: 17253876]

23. Osterman MT, Sandborn WJ, Colombel J- F, et al. Increased risk of malignancy with adalimumab combination therapy, compared with monotherapy, for Crohn's disease. Gastroenterology. 2014;146:941-949. [PubMed: 24361468] 


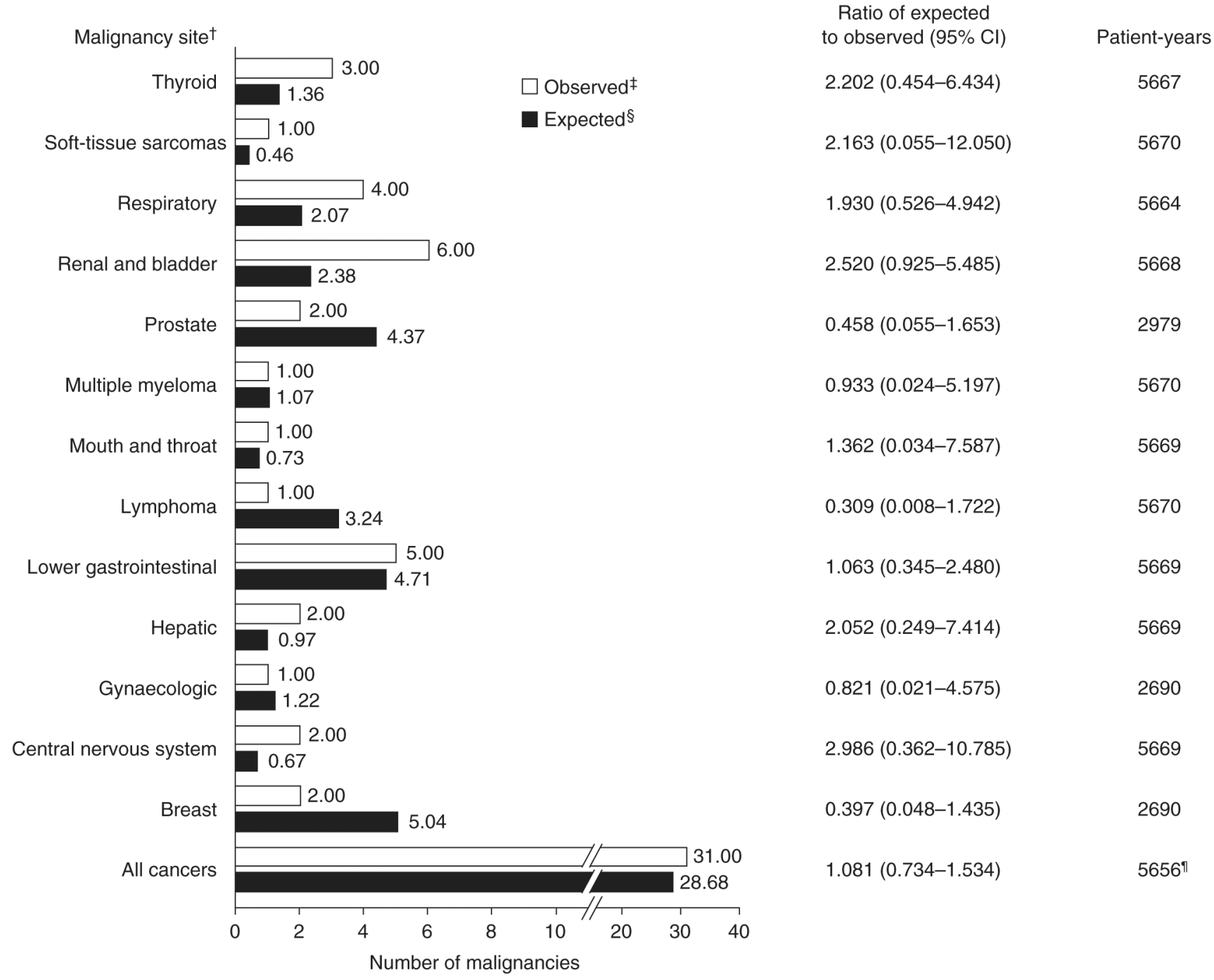

FIGURE 1.

Indirect standardisation of the numbers of GEMINI LTS study patients with malignancy events by anatomical site. Data shown exclude malignancies with a diagnosis reported prior to, or within $1 \mathrm{y}$ after starting treatment with vedolizumab and the following events: benign neoplasms, basal cell carcinomas, colon adenomas, haemangioma, neurilemmoma, nonmelanoma skin cancers and malignancies with diagnoses reported before vedolizumab initiation. CI, confidence interval; LTS, long-term safety. ${ }^{\dagger}$ Malignancies were identified using Medical Dictionary for Regulatory Activities version 20.0 System Organ Class term "neoplasms benign, malignant, and unspecified (including cysts and polyps)". Benign neoplasms, basal cell carcinoma, colon adenoma, haemangioma, neurilemmoma, all nonmelanoma skin cancers and malignancies with diagnoses reported before, or within $1 \mathrm{y}$ after, the start of vedolizumab treatment (ie those in patients who received placebo in the lead-in studies) were excluded. The corresponding follow-up time for excluded malignancies was not used in the analysis. + Patients with more than one malignancy at a single site were counted only once. ${ }^{\S}$ The expected number of patients with malignancies in the GEMINI LTS study was estimated by indirectly standardising against age- and sex-specific malignancy 
rates in patients with inflammatory bowel disease in Optum's Clinformatics Data Mart database. "ISurvival time was measured up to time of failure or end of study follow-up. As a result, the total number of patient-years is reduced vs values of each site individually 


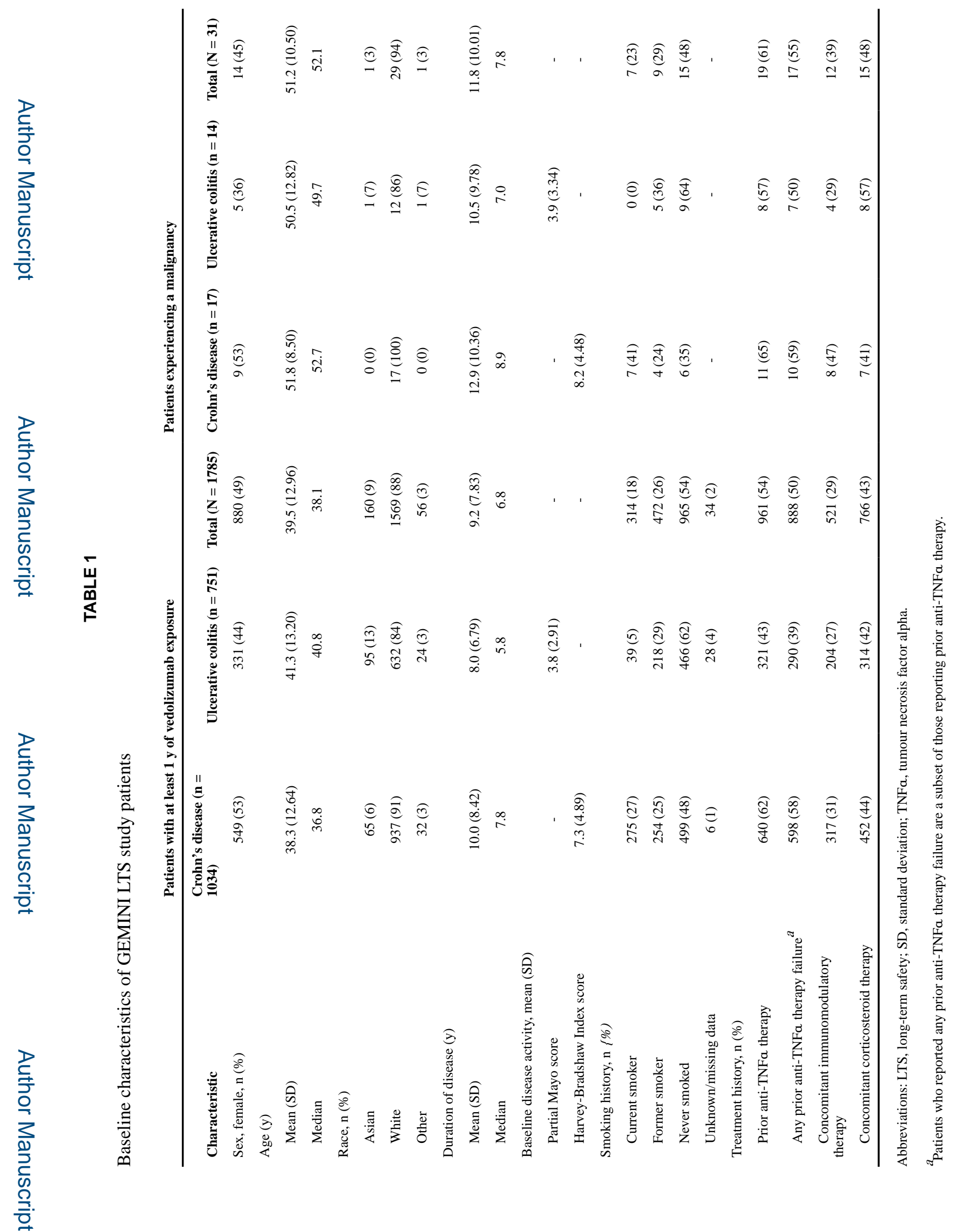

Aliment Pharmacol Ther. Author manuscript; available in PMC 2020 March 02. 


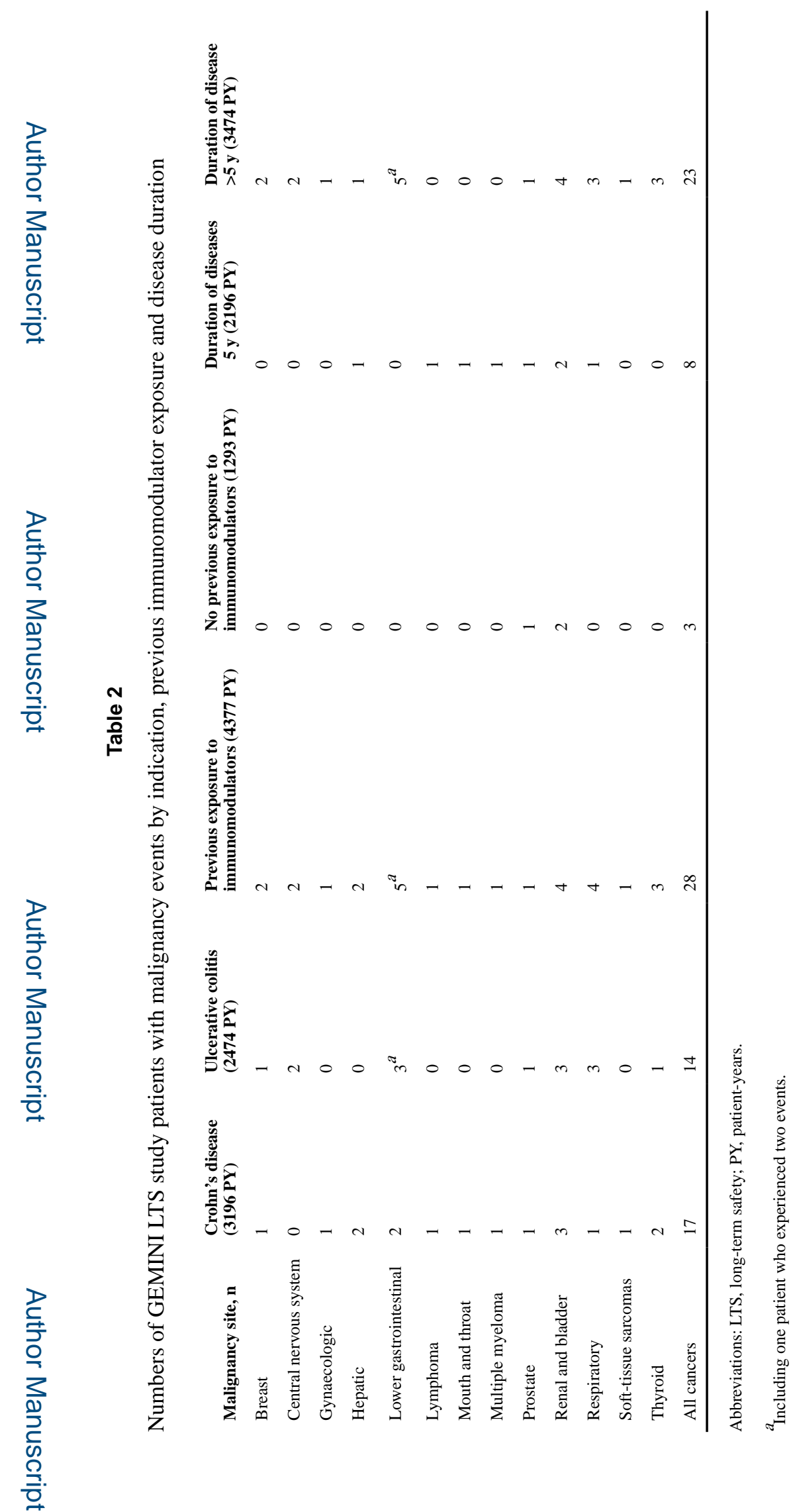

Aliment Pharmacol Ther. Author manuscript; available in PMC 2020 March 02. 


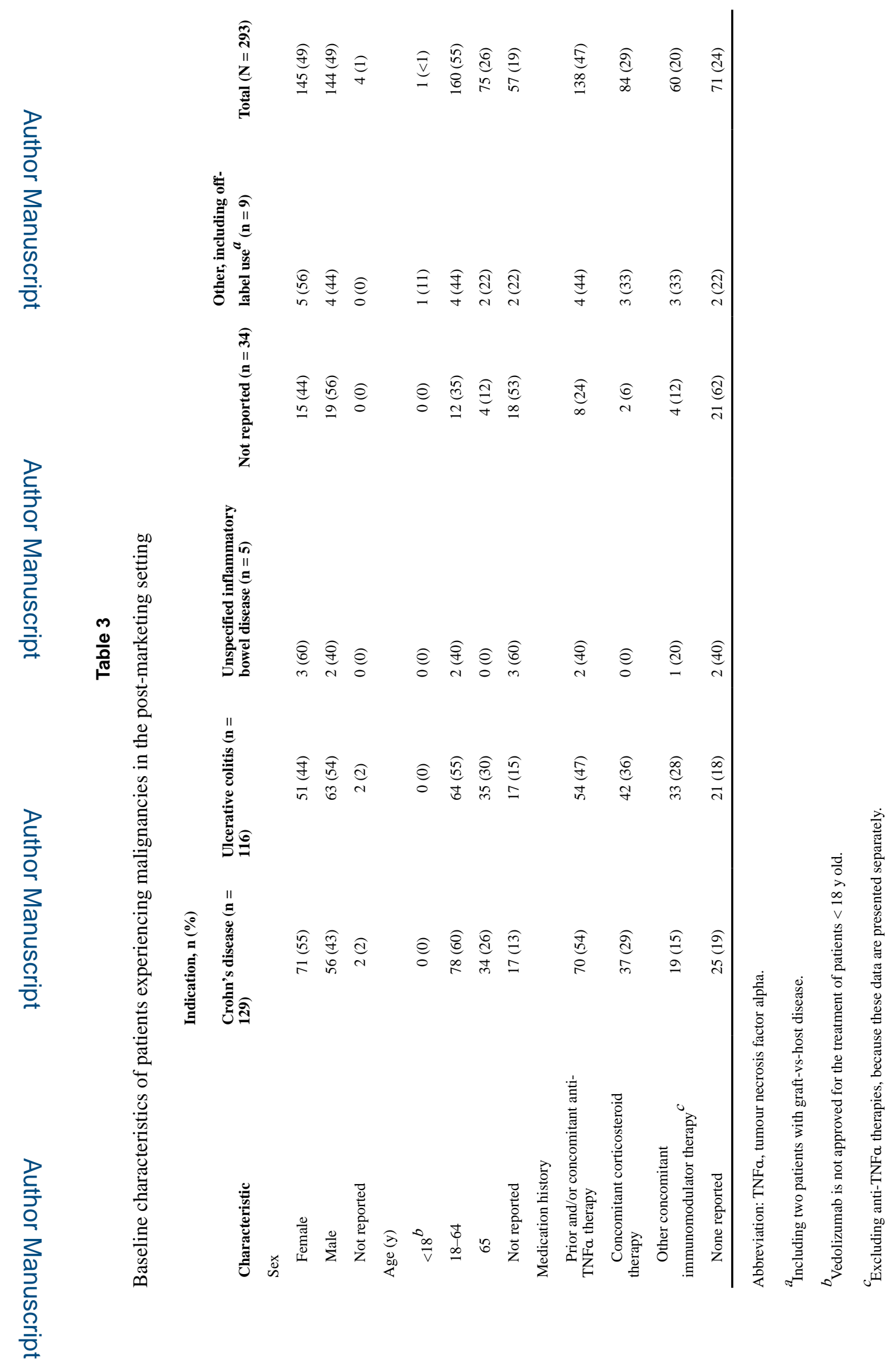

Aliment Pharmacol Ther. Author manuscript; available in PMC 2020 March 02. 